\title{
Síntesis de materiales catalíticos a partir de una arcilla natural para la valorización de alcoholes
}

\section{Synthesis of catalytic materials from a natural clay for the valorization of alcohols}

Presentación: 11/10/2019

Doctorando:

\section{Ema V. Sabre}

Centro de Investigación y Tecnología Química (CITeQ-UTN-CONICET). UTN-FRC

emasabre@gmail.com

\section{Directora:}

\section{Dra. Analía L. Cánepa}

\section{Co-Directora:}

\section{Dra. Sandra G. Casuscelli}

\begin{abstract}
Resumen
Se plantea el estudio de la oxidación de alcoholes a los correspondientes compuestos carbonílicos mediante procesos catalíticos sustentables, empleando tecnologías que apunten a un bajo impacto ambiental, para evitar la generación de compuestos potencialmente contaminantes, optimizando la selección de reactivos y utilizando catalizadores heterogéneos. Para ello se utiliza una arcilla natural, de bajo costo debido a su abundancia en la naturaleza, como punto de partida para sintetizar materiales con buenas propiedades catalíticas. Así, mediante el proceso de pilareado las arcillas se transforman en sólidos con alta porosidad y superficie específica, denominándose PILC, las cuales pueden ser modificadas posteriormente con iones metálicos. Estos materiales permiten transformar diferentes compuestos químicos, como por ejemplo los alcoholes, de manera eficiente y selectiva en condiciones suaves, a aldehídos, epóxidos, etc. Estos productos pueden utilizarse como intermediarios en la industria de Química Fina en la obtención de productos farmacéuticos, colorantes, agroquímicos, aditivos plastificantes, resinas y fragancias.
\end{abstract}

Palabras claves: Arcillas pilareadas, Química fina, Oxidación selectiva, Alcoholes, Procesos sustentables.

\begin{abstract}
The study of the oxidation of alcohols to the corresponding carbonyl compounds is proposed through sustainable catalytic processes, using technologies that point to a low environmental impact, to avoid the generation of potentially polluting compounds, optimizing the selection of reagents and using heterogeneous catalysts. For this, a low-cost natural clay is used due to its abundance in nature, as a starting point to synthesize materials with good catalytic properties. Thus, through a pillared process the clays are transformed into solids with high porosity and specific surface, denominating PILC, which can be modified later with metal ions. These materials allow different chemical compounds, such as alcohols, to be transformed efficiently and selectively under mild conditions, to aldehydes, epoxides, etc. These products can be used as intermediaries in the Fine Chemistry industry: obtaining pharmaceutical products, dyes, agrochemicals, plasticizer additives, resins and fragrances.
\end{abstract}

Keywords: Pillared clays, Fine chemistry, Selective oxidation, Alcohols, Sustainable processes. 


\section{Introducción}

El desarrollo de sistemas catalíticos sustentables se ha convertido en los últimos años en el objetivo de numerosas investigaciones. En este sentido, una alternativa importante es el uso de arcillas naturales para la síntesis de materiales con posibles aplicaciones catalíticas. Las arcillas son interesantes debido a su bajo costo y su gran abundancia en la naturaleza. En particular, en Argentina se encuentran importantes yacimientos de arcilla en las provincias de Córdoba, San Juan y Neuquén. Las arcillas son filosilicatos ("filo" significa hoja), compuestas por mezclas de minerales arcillosos, cenizas volcánicas, sílices y carbonatos; se clasifican en tres grupos principales: caolín, micas y esmectitas. A su vez, la esmectita conforma una familia de arcillas compuesta de: montmorillonita, saponita, vermiculita, nontronita, hectorita, volkonskoita, sauconita, y beidellita. La montmorillonita es probablemente una de las arcillas minerales más estudiadas, ya que las propiedades fisicoquímicas y mecánicas de la misma pueden modificarse mediante procedimientos simples convirtiéndolas en materiales efectivos para adsorción y catálisis [1,2].

La estructura de la montmorillonita consiste en una capa de alúmina octaédrica $\left[\mathrm{Al}_{2}(\mathrm{OH})_{6}\right]$ intercalada entre dos capas de silicatos tetraédricos $\left[\mathrm{SiO}_{4}\right]^{4-}$. En presencia de moléculas de agua, el espacio entre capas se incrementa, lo que facilita el intercambio de los cationes que se encuentran en la intercapa, generalmente $\mathrm{Na}^{+}, \mathrm{Ca}^{2+} \mathrm{y} \mathrm{Mg}^{2+}$. $\mathrm{Las}$ cargas negativas en la capa octaédrica se originan de la sustitución isomorfa de $\mathrm{Al}^{3+}$ por especies de $\mathrm{Mg}^{2+}$. Si la montmorillonita se encuentra deshidratada, los cationes intercambiables se encuentran dentro de las cavidades hexagonales de la capa de sílice, pero tras la hidratación, los cationes se mueven hacia el espacio de intercapas [1]. Debido a su capacidad de intercambio catiónico (CIC) y propiedades expandibles [3], la eficiencia catalítica de las montmorillonitas se puede mejorar transformándolas en sólidos con alta microporosidad y superficie específica [4]. Los cationes que poseen en su intercapa pueden ser intercambiados por cationes provenientes de una solución externa. Una amplia variedad de especies policatiónicas inorgánicas pueden ser insertadas en la intercapa por intercambio catiónico. Posteriormente estas especies policatiónicas se transforman en óxidos metálicos mediante tratamiento a altas temperaturas, por deshidratación y dehidroxilación, formando pilares rígidos que se enlazan a las capas de silicatos, aumentando la distancia de separación entre ellas. Así, se obtienen las denominadas arcillas pilareadas (PILCs), las cuales poseen una buena distribución de microporos y mesoporos, alta área específica y estabilidad térmica, además de sitios ácidos de Brönsted y Lewis. Las propiedades ácidas y redox de estos materiales los convierte en sólidos muy eficientes en diversos campos como: refinamiento de petróleo, química fina, catálisis ambiental y fotocatálisis [5-9]. Hasta el momento se han reportado diferentes especies policatiónicas como precursoras de los pilares de la arcilla, los cuales contienen diferentes metales tales como: Al, Fe, Ti, Ce y Zr. Así, se acostumbra a nombrar a las arcillas pilareadas como X-PILC, donde X hace referencia al metal utilizado como precursor del pilar. En particular, las Ti-PILC, con alta estabilidad térmica e hidrotérmica, gran tamaño de poro, alta área específica y valores de acidez y buena capacidad de fotooxidación [7] pueden ser aplicadas eficientemente como catalizadores en la industria química fina y como fotocatalizadores en catálisis ambiental [10].

La actividad catalítica en las arcillas pilareadas puede estar asociada intrínsecamente a los óxidos metálicos que actúan como pilares y/o a especies activas que se incorporan [11]. Los métodos de incorporación del componente activo pueden ser: síntesis directa, donde el componente activo se agrega a la solución pilareante, y se pone en contacto con la arcilla bajo condiciones apropiadas [12], o post-síntesis, mediante impregnación e intercambio iónico $[13,14]$. En este último, se sintetiza la PILC y luego el componente activo se incorpora desde una solución externa, bajo condiciones adecuadas. El método de impregnación permite obtener una mayor estabilidad térmica del catalizador, logrando una distribución homogénea de los componentes activos mayoritariamente en la superficie. Estos materiales, a diferencia de otros catalizadores convencionales, presentan considerables ventajas que contribuyen a la "Química Verde" [15] tales como bajo costo, facilidad de manejo y posibilidad de reutilización. Las PILCs pueden emplearse como catalizadores heterogéneos para transformar compuestos orgánicos de manera eficiente y selectiva en condiciones suaves (a temperatura $<100 \stackrel{\circ}{\circ}$ ), que permitan el ahorro de energía. En este sentido, la oxidación de alcoholes a los correspondientes compuestos carbonílicos es una transformación orgánica importante en la industria de química fina, debido a que pueden utilizarse como intermediarios en la obtención de productos farmacéuticos, colorantes, agroquímicos, aditivos plastificantes, resinas y fragancias [16-18]. Entre los intermediarios más importantes se encuentran los aldehídos, como el benzaldehído (BzH), obtenido de la oxidación del alcohol bencílico (BzOH). Se utiliza en perfumería, cosmética, en industrias alimentarias y como precursor de pinturas y de otros compuestos orgánicos [5,13,14].

Además del catalizador heterogéneo, otro aspecto importante a tener en cuenta en las reacciones de oxidación es el tipo de oxidante que se utiliza. En este sentido, el empleo de las PILCs evita el uso de oxidantes fuertes como el clorocromato de piridinio (PCC), el dicromato de piridinio (PDC), $\mathrm{KMnO}_{4}, \mathrm{MnO}_{2}, \mathrm{Na}_{2} \mathrm{Cr}_{2} \mathrm{O}_{7}, \mathrm{CrO}_{3}$, entre otros [19,20]. Estos oxidantes son caros, peligrosos o tóxicos y habitualmente requieren disolventes, lo que genera una cantidad 
importante de residuos inorgánicos que luego deberán ser tratados, con las desventajas que esto presenta tanto desde el punto de vista económico como medioambiental. En su reemplazo, oxidantes como oxígeno, peróxido de hidrógeno, terbutil hidroperóxido (TBHP), entre otros, están siendo cada vez más elegidos. En particular, el peróxido de hidrógeno se considera un oxidante limpio y seguro, barato y amigable con el medio ambiente, ya que sólo se obtiene agua y oxígeno como subproductos.

Por todo lo expuesto se plantea que es factible la obtención de compuestos intermediarios para la síntesis de productos de alto valor agregado, a partir de la oxidación de alcoholes orgánicos, como el alcohol bencílico y el etanol, empleando oxidantes limpios y catalizadores heterogéneos como las arcillas pilareadas. Así, se pretende que la obtención de compuestos de interés industrial mediante la utilización de tecnologías limpias, empleando arcillas naturales de bajo costo para la síntesis de catalizadores, puedan realizar contribuciones desde el punto de vista económico, social y ambiental al desarrollo de sistemas catalíticos sostenibles.

\section{Experimental}

\section{Síntesis de los catalizadores}

Se utilizó montmorillonita proveniente de la provincia de San Juan. Este material se intercambió con iones $\mathrm{Na}^{+}$, denominándose luego del intercambio como Na-mont. La solución pilareante se preparó adicionando la cantidad de $\mathrm{Ti}\left(\mathrm{OC}_{4} \mathrm{H}_{9}\right)_{4}$ (Fluka) necesaria, a una solución de $\mathrm{HCl} 6 \mathrm{M}$ bajo agitación vigorosa, para conseguir $10 \mathrm{mmol}$ de Ti/g de arcilla. Se adicionó por goteo a 0,5 L de una suspensión de Na-mont que contenía $8 \mathrm{~g} / \mathrm{L}$. La fracción sólida se separó por centrifugación, y se lavó con agua destilada hasta quedar libre de iones $\mathrm{Cl}$. El sólido seco se calcinó a $450^{\circ} \mathrm{C}$ durante $2 \mathrm{~h}$, denominándose Ti-PILC. Posteriormente, se incorporó el vanadio en el soporte Ti-PILC mediante impregnación húmeda. Para ello, se agregó por goteo una solución acuosa con la cantidad necesaria de sulfato de vanadilo hidratado (99\%) (Aldrich) como fuente de metal, para obtener un $\mathrm{x} \%$ en peso del precursor metálico en el material, el solvente se eliminó en un evaporador rotatorio. Finalmente, el sólido seco se calcinó a $450{ }^{\circ} \mathrm{C}$ durante $3 \mathrm{~h}$ bajo flujo de aire, denominándose $\mathrm{V}(\mathrm{x} \%)$ /Ti-PILC, donde $\mathrm{x}$ indica el porcentaje en peso de vanadio en el soporte. Se sintetizaron materiales con los siguientes con tenidos de V: $0,1,0,5$ y $1 \% \mathrm{p} / \mathrm{p}$.

\section{Caracterización de los catalizadores}

Los materiales sintetizados fueron caracterizados por Difracción de rayos X, los patrones de difracción se realizaron en un difractómetro D8 Advance BRUKER con radiación $\mathrm{Cu} K \alpha(\lambda=1.5418 \AA$ ) en el rango de $2 \theta$ entre 2 y $70^{\circ}$. El área superficial (ABET), el volumen total de poros (Vtp) y el volumen de microporos (Vup) fueron determinados de las isotermas de adsorción-desorción de N2 obtenidas a -196 ${ }^{\circ} \mathrm{C}$ usando un equipo Gemini V2.00 Micromeritics. Los espectros de UV-vis con Reflectancia Difusa (UV-vis-RD) se realizaron para estudiar el entorno de coordinación y la posición de las especies metálicas en las matrices sólidas. Para tal fin se utilizó un espectrómetro Jasco V650 equipado con esfera integradora tipo RSA-PE-20, en el rango de 200-900 nm.

\section{Evaluación catalítica}

La actividad catalítica de los materiales sintetizados se evaluó en reacciones de oxidación de BzOH (Fluka > 95\%), con acetonitrilo (Sintorgan 99,5\%) como solvente y $\mathrm{H}_{2} \mathrm{O}_{2}$ (Riedel de Haen $35 \% \mathrm{p} / \mathrm{p}$ ) como oxidante. Las mismas se llevaron a cabo en un reactor de vidrio tipo batch, equipado con un agitador magnético y un condensador a reflujo sumergido en un baño a $70{ }^{\circ} \mathrm{C}$ durante $5 \mathrm{~h}$. El avance de la reacción se siguió tomando muestras a diferentes tiempos. Las alícuotas se filtraron y analizaron por cromatografía gaseosa utilizando un cromatógrafo Agilent 7820 con Columna Capilar HP-1 y detector FID. El $\mathrm{H}_{2} \mathrm{O}_{2}$ remanente fue determinado por titulación iodométrica. La conversión (\% mol) del $\mathrm{BzOH}$ y del $\mathrm{H}_{2} \mathrm{O}_{2}$, la selectividad (\%) a los productos de reacción y el rendimiento (\%) se calcularon según las Ecuaciones (1), (2) y (3).

Conversión $(\%)=\frac{\text { mmoles de productos oxidados }}{\text { mmoles reactivo iniciales }} \times 100$

Selectividad $_{i}(\%)=\frac{\text { mol producto }_{i}}{\text { mol productos totales }} \times 100$

Rendimiento $_{i}(\%)=$ Conversión $_{(\%)} \times$ Selectividad $_{i}$

\section{Resultados}




\section{Caracterización de los catalizadores}

La Figura 1, presenta los DRX de los materiales: Na-mont, Ti-PILC, V(0,1\%)/Ti-PILC, V(0,5\%)/Ti-PILC y V(1\%)/TiPILC en el rango de $2 \theta$ entre 2 y $20^{\circ}$. El espectro de Na-mont muestra una reflexión a $2 \theta \approx 7,2$, asignada al espaciamiento basal d001 $=12,27 \AA$, dicho valor representa la distancia entre dos capas de la arcilla. Luego del proceso de pilareado, la reflexión 001 aparece a un valor menor de $2 \theta \approx 5,2$, incrementando el espaciamiento basal de las capas de la arcilla a d001 = $17 \AA$, originado por los policationes de Ti con diferentes grados de polimerización. La incorporación de $\mathrm{V}$ en diferentes contenidos, disminuye el espaciamiento basal a valores próximos a $16 \AA$, posiblemente debido al desorden estructural generado en la arcilla durante el proceso de impregnación del $V$ en la Ti-PILC [21-23].

En la Figura 2 se presentan los DRX de Na-mont, Ti-PILC, V(0,1\%)/Ti-PILC, V(0,5\%)/Ti-PILC y V(1\%)/Ti-PILC, en el rango de $2 \theta$ entre 24 y $70^{\circ}$.

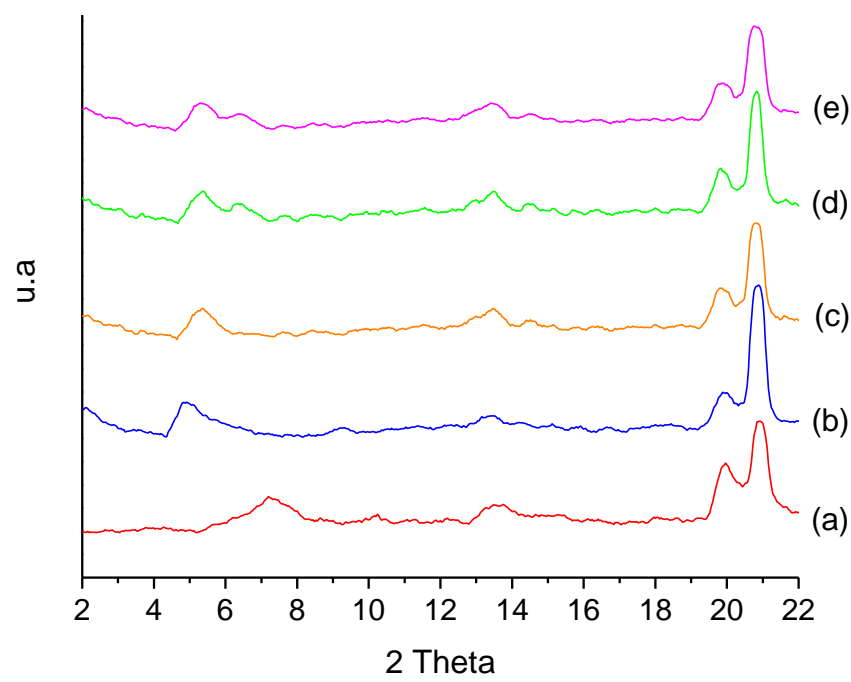

Figura 1. Espectros de DRX: (a) Na-mont, (b) TiPILC, (c) V(0,1\%)/Ti-PILC, (d) V(0,5\%)/Ti-PILC, (e) $\mathrm{V}(1 \%) / \mathrm{Ti}-\mathrm{PILC}$.

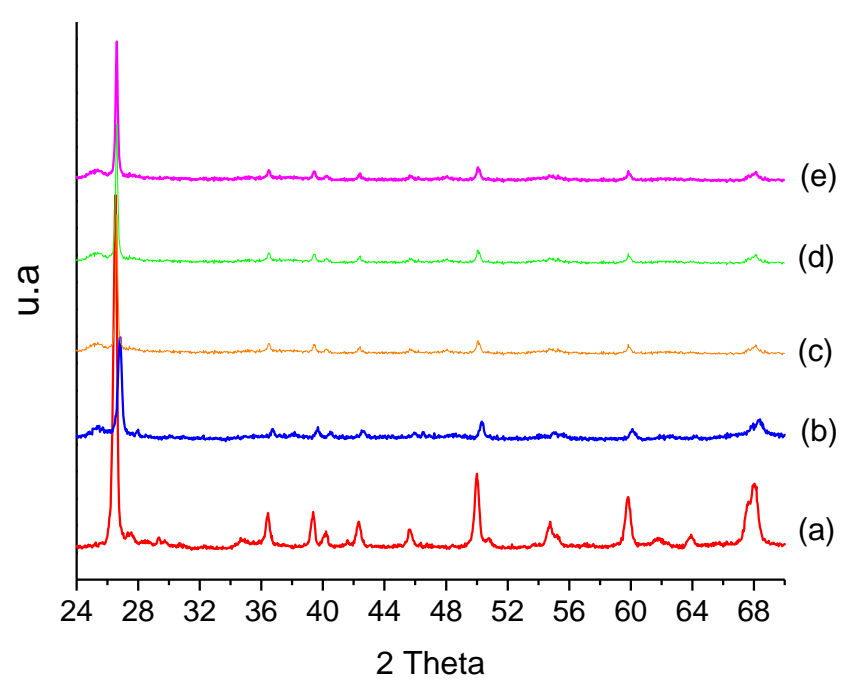

Figura 2. Espectros de DRX: (a) Na-mont, (b) TiPILC, (c) V(0,1\%)/Ti-PILC, (d) V(0,5\%)/Ti-PILC, (e) V(1\%)/Ti-PILC.

La muestra Na-mont presenta un pico aproximadamente a 26,7ㅇ característico del $\mathrm{SiO}_{2}$ (cuarzo), el mismo decrece notablemente para Ti-PILC y los materiales con V [22]. Para los materiales pilareados, se observa además una reflexión a $2 \theta \approx 25$ o, 2 asignada a la fase anatasa del $\mathrm{TiO}_{2}$. En los materiales que poseen $\mathrm{V}$, no se observa la fase cristalina de $\mathrm{V}_{2} \mathrm{O}_{5}$, lo que sugiere que el vanadio presente se encuentra en forma amorfa o muy disperso sobre la superficie de Ti-PILC [23,24].

Los resultados de las propiedades texturales de los catalizadores sintetizados en este trabajo se presentan en la Tabla 1.

Tabla 1: Propiedades texturales de los catalizadores sintetizados.

\begin{tabular}{lcccc}
\hline \multicolumn{1}{c}{ Catalizador } & $\begin{array}{c}\text { Contenido de } \mathrm{V} \\
\text { (\% en peso) }\end{array}$ & $\begin{array}{c}\mathrm{A}_{\mathrm{BET}^{\mathrm{a}}} \\
\left(\mathrm{m}^{2} / \mathrm{g}\right)\end{array}$ & $\begin{array}{c}\mathrm{Vtp}^{\mathrm{b}} \\
\left(\mathrm{cm}^{3} / \mathrm{g}\right)\end{array}$ & $\begin{array}{c}\mathrm{V} \mu \mathrm{p}^{\mathrm{c}} \\
\left(\mathrm{cm}^{3} / \mathrm{g}\right)\end{array}$ \\
\hline Na-mont & - & 31 & 0,055 & 0,004 \\
Ti-PILC & - & 105 & 0,131 & 0,003 \\
$\mathrm{~V}(0,1 \%) /$ Ti-PILC & 0,1 & 89 & 0,142 & 0,002 \\
$\mathrm{~V}(0,5 \%) /$ Ti-PILC & 0,5 & 78 & 0,137 & 0,001 \\
$\mathrm{~V}(1 \%) /$ Ti-PILC & 1 & 70 & 0,125 & $<0,001$ \\
\hline
\end{tabular}

a Área específica calculada a partir de la isoterma de adsorción de nitrógeno utilizando la ecuación de BET.

b Volumen total de poros calculado a partir de la isoterma de adsorción de nitrógeno.

c Volumen de microporos calculado a partir de la isoterma de adsorción de nitrógeno.

Como puede observarse, la superficie específica y porosidad de Ti-PILC son claramente superiores que las del material de partida (Na-mont). Esto refleja la formación de los pilares que expanden las capas de las arcillas. Luego de la adición de V en Ti-PILC, se observó que tanto el área específica como el volumen de microporos disminuyen. Este hecho se atribuye al bloqueo de los poros más pequeños por las especies de $\mathrm{V}$ depositadas. 
Se empleó la técnica de UV-Vis RD para dilucidar el entorno de coordinación de las especies metálicas presente en los materiales bajo estudio. Los espectros obtenidos se presentan en la Figura 3. El espectro de la arcilla sódica (Namont) muestra una banda centrada alrededor de $250 \mathrm{~nm}$, asignada a la transferencia de carga entre los ligandos de oxígeno y los iones de $\mathrm{Fe}^{3+}$ presentes en la estructura de la arcilla $[21,25]$.

El espectro de Ti-PILC presenta una banda alrededor de $210 \mathrm{~nm}$, la cual se puede atribuir a las especies de $\mathrm{Ti}^{4+}$ en una coordinación tetraédrica [25]. Además se observa otra banda centrada alrededor de $260 \mathrm{~nm}$, asignada a especies de $\mathrm{Ti}^{4+}$ aisladas en coordinación octaédrica. La banda que se extiende entre 290-320 nm se asigna a la fase anatasa del $\mathrm{TiO}_{2}$.

Por otra parte, los materiales modificados con vanadio presentaron una leve disminución en la intensidad de las principales bandas de absorción respecto a Ti-PILC, debido probablemente a la interrupción de las cadenas Ti-O-Ti por la incorporación de especies de vanadio [22].

Es conocido que materiales modificados con vanadio presentan absorción en la zona entre 200-600 nm. Así para el material con mayor contenido de vanadio se observa una absorción localizada entre 350-500 nm asignada a iones vanadio penta o hexacoordinados formando nanoclusters en la estructura debido a la oligomerización incipiente de especies de vanadio [25]. Además una banda a $260 \mathrm{~nm}$ solapada a la absorción de Ti-PILC, debe ser considerada debido a la presencia de iones de vanadio aislados en coordinación tetraédrica con oxígenos de la red.

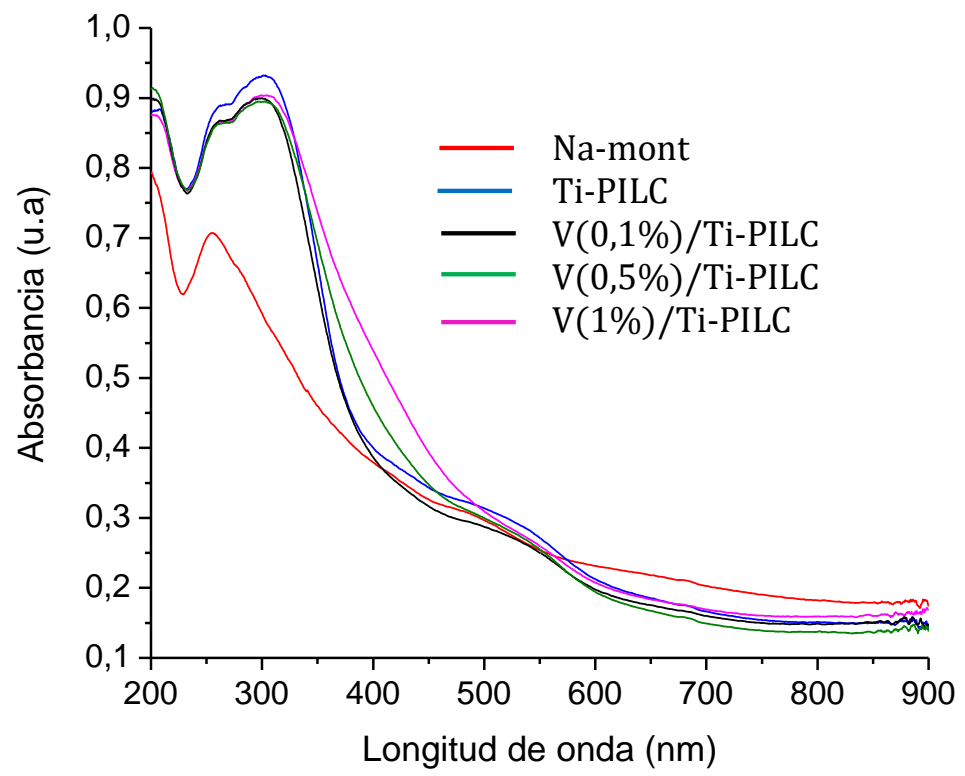

Figura 3. Espectros UV-vis-RD para: Na-mont, Ti-PILC, V(0,1\%)/Ti-PILC, V(0,5\%)/Ti-PILC y V(1\%)/Ti-PILC.

Evaluación catalítica

Los resultados obtenidos en la oxidación del $\mathrm{BzOH}$ con los materiales sintetizados se presentan en la Tabla 2.

Tabla 2: Oxidación del BzOH con los catalizadores sintetizados a 5 h de reacción.

\begin{tabular}{|c|c|c|c|c|c|c|}
\hline \multirow{2}{*}{ Catalizador } & \multirow{2}{*}{$\begin{array}{c}\text { Conversión } \\
(\% \mathrm{~mol}) \mathrm{de} \\
\mathrm{BzOH}\end{array}$} & \multirow{2}{*}{$\begin{array}{c}\text { Conversión } \\
(\% \mathrm{~mol}) \text { de } \\
\mathrm{H}_{2} \mathrm{O}_{2}\end{array}$} & \multirow{2}{*}{$\begin{array}{l}\text { Rendimiento } \\
\text { a BzH (\%) }\end{array}$} & \multicolumn{3}{|c|}{ Selectividad (\%) } \\
\hline & & & & $\mathrm{BzH}$ & BzA & BzB \\
\hline Ti-PILC & 4,02 & 65,67 & 3,82 & 95,10 & 2,71 & 2,19 \\
\hline V(0,1\%)/Ti-PILC & 7,50 & 76,46 & 7,08 & 94,45 & 3,05 & 2,50 \\
\hline V(0,5\%)/Ti-PILC & 10,87 & 95,49 & 10,29 & 94,72 & 4,15 & 1,13 \\
\hline V(1\%)/Ti-PILC & 8,62 & $93,58^{*}$ & 7,92 & 91,91 & 6,29 & 1,80 \\
\hline
\end{tabular}

Condiciones de reacción: 9,11 mmol de $\mathrm{BzOH}, 91,16 \mathrm{mmol}$ de $\mathrm{AcN}, 2,27 \mathrm{mmol}$ de $\mathrm{H}_{2} \mathrm{O}_{2}$ y $54 \mathrm{mg}$ de catalizador. Temperatura de reacción: $70 \stackrel{\circ}{\circ}$. ${ }^{*}$ Agregado de $\mathrm{H}_{2} \mathrm{O}_{2}$ a la hora de reacción.

Ácido benzoico (BzA), Benzoato de bencilo (BzB)

El material de partida Na-Mont resultó inactivo para la reacción bajo estudio, por su parte la incorporación del Ti en la arcilla mejoró la actividad catalítica, alcanzando una conversión del 4,02\% del $\mathrm{BzOH}$ a las $5 \mathrm{~h}$ de reacción. A su vez la incorporación de $\mathrm{V}$ en Ti-PILC produjo un importante incremento de la conversión (\% mol) del BzOH, el cual aumenta con el contenido de $\mathrm{V}$ hasta un valor máximo de aproximadamente $11 \%$ para una carga del $0,5 \%$ en peso de 
V. Un incremento posterior en la carga no resulta en un aumento de la actividad. Por otra parte, el consumo de $\mathrm{H}_{2} \mathrm{O}_{2}$ aumentó con el contenido de V, observándose un consumo casi total (93\%) a la hora de reacción para V(1\%)/TiPILC, lo cual hizo necesario un agregado adicional del oxidante para reestablecer la concentración inicial de 0,38 mmol de $\mathrm{H}_{2} \mathrm{O}_{2} / \mathrm{mL}$ de solución reaccionante. Este comportamiento podría ser asociado a los nanoclusters de vanadio observados por UV-vis-RD para las muestra con mayor carga, así esta especie estaría involucrada en la descomposición del $\mathrm{H}_{2} \mathrm{O}_{2}$ haciendo necesario el agregado adicional. Además, los buenos resultados obtenidos con la muestra con $0,5 \% \mathrm{~V}$ podrían ser relacionados con la presencia de iones de vanadio aislados en coordinación tetraédrica que favorecería el rendimiento a benzaldehído. Es importante destacar que la relación molar de $\mathrm{BzOH} / \mathrm{H}_{2} \mathrm{O}_{2}$ empleada fue 4:1, es decir el $\mathrm{H}_{2} \mathrm{O}_{2}$ es el reactivo limitante, con lo cual la conversión máxima del BzOH (\% mol) que puede obtenerse es del $25 \%$.

En cuanto a los productos de reacción obtenidos, el mayoritario fue el $\mathrm{BzH}$, obteniéndose además BzA y BzB como subproductos. El catalizador que presentó el mayor rendimiento a BzH fue el V(0,5\%)/Ti-PILC con una selectividad de $94,72 \%$. No obstante, todos los materiales modificados con metales de transición mostraron una elevada especificidad al producto deseado.

\section{Conclusiones}

Fue posible incrementar el área específica y espaciamiento basal de la arcilla natural mediante el proceso de pilareado con $\mathrm{Ti}$, además se pudo incorporar un segundo ión metálico como el $\mathrm{V}$ por impregnación húmeda, lo cual incrementó su performance catalítica en la reacción de oxidación de alcohol bencílico a benzaldehído.

El catalizador V(0,5\%)/Ti-PILC fue el más activo, logrando una conversión del 10,87\% mol del BzOH a las $5 \mathrm{~h}$ de reacción, y una selectividad al producto deseado del $94,72 \%$. Estos resultados podrían relacionarse con la presencia de iones de vanadio aislados en coordinación tetraédrica que favorecería el rendimiento a benzaldehído. En cambio, la actividad catalítica disminuyó para el material con el mayor contenido de V, observándose por UV-vis-RD la presencia de nanoclusters de óxidos de $\mathrm{V}$, responsables de la descomposición del $\mathrm{H}_{2} \mathrm{O}_{2}$ y por consiguiente una menor conversión del BzOH.

\section{Referencias}

[1] Nagendrappa G. (2002). Resonance, 7, 64-71.

[2] Kaur N., Kishore D., Chem J. (2012). Pharm. Res., 4, 991-1015.

[3] Jagtap N., Ramaswamy V. (2006). Appl. Clay Sci., 33, 89-98.

[4] Andrea De Leon M., De Los Santos C., Latronica L., Cesio A. M., Volzone C., Castiglioni J., Sergio M. (2014). Chem. Eng. J., 241, 336-343.

[5] Arfaoui J., Ghorbel A., Petitto C., Delahay G. $\quad$ (2017) J. Chem. $\quad$ Eng., doi: http://dx.doi.org/10.1016/j.cej.2017.02.046.

[6] Lyu X., Mao H., Zhu K., Kong Y., Kobayashi M. (2017). Microporous Mesoporous Mater., 252, 1-9.

[7] Li J., Hu M., Zuo S., Wang X. (2018). Opinion in Chem. Eng., 20, 93-98.

[8] Zang S., Zhang G., Qiu W., Song L., Zhang R., He H. (2016). Chinese J. Catal., 37, 888-897.

[9] Tomul F., Turgut Basoglu F., Canbay H. (2016). Appl. Surf. Sci., 360, 579-593.

[10] Zuo S., Ding M., Tong J., Feng L., Qi C. (2015). Appl. Clay Sci., 105-106, 118-123.

[11] Gatica J. M., Castiglioni J., de los Santos C., Yeste M. P., Cifredo G., Torres M. (2017). Catal. Today, 296, 84-94.

[12] Caudo S., Centi G., Genovese C., Perathoner S. (2007). Appl. Catal. B: Environ., 70, 437-446.

[13] Dorado F., de Lucas A., García P. B., Romero A., Valverde J. L. (2006). Appl. Catal. A: Gen., 305, 189-196.

[14] Valverde J. L., de Lucas A., Sánchez P., Dorado F., Romero A. (2003). Appl. Catal. B: Environ., 43, 43-56.

[15] Anastas P. T., Kirchhoff M. M., Williamson T. C. (2001). Appl. Catal. A: Gen., 221, 3-13.

[16] Tojo G., Fernández M. (2006). Ed. Springer, ISBN 978-0-387-25725-9

[17] Davis S. E., Ide M. S., Davis R. J. (2013). Green Chem., 15, 17-45.

[18] Dormán G., Prestwich G. D. (1994). Biochemistry, 33, 5661-5673

[19] Holum J. R., Org. J. (1961). Chem., 26, 4814-4816.

[20] Dutta D., Phukan A., Kumar Dutta D. (2018). Mol. Catal., 451, 178-185.

[21] Arfaoui J., Khalfallah Boudali L., Ghorbel A., Delahay G. (2009). Catal. Today, 142, 34-238.

[22] Chen J., Liu X., Li G., Nie X., An T., Zhang S., Zhao H. (2011). Catal. Today, 164, 364-369.

[23] Chae H., Nam I., Ham S., Hong S. (2004). Appl. Catal. B: Environ., 53, 117-126.

[24] Zang S., Zhang G., Qiu W., Song L., Zhang R., He H. (2016). Chinese J. Catal., 37, 888-897.

[25] Dali A., Rekkab-Hammoumraoui I., Choukchou-Braham A., Bachir R. (2014) RSC Advances Doi: $0.1039 / \mathrm{x} 0 \mathrm{xx} 00000 \mathrm{x}$. 